\title{
Grandparental and Parental Obesity Influences on Childhood Overweight: Implications for Primary
} Care Practice

\author{
Matthew M. Davis, MD, MAPP, Katherine McGonagle, PhD, Robert F. Schoeni, PhD, \\ and Frank Stafford, PhD
}

Background: Community-based studies have suggested a multigenerational pattern of obesity affecting children's risk of overweight, but no national data have substantiated such a pattern. Our objective was to examine the prevalence of overweight [body mass index (BMI) $\geq 95$ th percentile for age and sex] among children aged 5 to 19 in a national sample, stratified by the obesity status of their parents and grandparents.

Methods: We used a secondary analysis of the Panel Study of Income Dynamics, Child Development Supplement, a multigenerational, genealogical, prospective cohort study of the US population. Self-report height and weight data from adults and measured height and weight data for children were used to calculate BMI. The prevalence of child overweight was calculated for different possible combinations of parental and grandparental BMI status, including missing status.

Results: The sample included 2591 children aged 5 to 19 years, for whom parental BMI data were available for $94 \%$ and grandparental BMI data were available for $61 \%$. Prevalence of childhood overweight (18.6\%) in the sample was comparable with contemporaneous measured national data from other sources. Among children with normal-weight parents and normal-weight grandparents, $7.9 \%$ were overweight. In contrast, among children with overweight parents (BMI 25-29.9) and normal-weight grandparents, $17.9 \%$ were overweight, and among children with obese parents (BMI $\geq 30$ ) and normalweight grandparents, 31.9\% were overweight $(P<.0001)$. Importantly, when parents were normal weight, if grandparents were obese, then the prevalence of child overweight was $17.4 \%(P<.0001)$. The prevalence of child overweight was similarly elevated $(16.4 \%)$ when parents were normal weight and grandparental BMI was missing.

Conclusions: This is the first national study to find an association of child weight status with grandparental obesity, distinct from parental obesity. Primary care physicians may find it helpful to consider grandparents' weight status in judging risk of childhood overweight for their patients, especially when parents' weight is normal. (J Am Board Fam Med 2008;21:549-54.)

Obesity is associated with a multitude of health problems faced by primary care physicians, ranging from type 2 diabetes and heart disease to hyperlipidemia, asthma, and obstructive sleep apnea. According to the most recent national data regarding

This article was externally peer reviewed.

Submitted 31 May 2007; revised 5 June 2008; accepted 17 June 2008.

From the Child Health Evaluation and Research Unit, Division of General Pediatrics, and the Division of General Internal Medicine, University of Michigan Medical School (MMD); Gerald R. Ford School of Public Policy (MMD, RFS); and Institute for Social Research (KM, RFS, FS), University of Michigan, Ann Arbor.

Funding: none.

Conflict of interest: none declared.

Corresponding author: Matthew M. Davis, MD, MAPP, University of Michigan, 300 NIB, 6C23, Ann Arbor, MI 48109-5456 (E-mail: mattdav@med.umich.edu). body mass index (BMI; calculated as weight in kilograms divided by the square of height in meters), the prevalence of overweight (defined as $\mathrm{BMI} \geq 95$ th percentile for age and sex) has reached $18 \%$ of children aged 6 to 19 years, and the prevalence of obesity (BMI $\geq 30 \mathrm{~kg} / \mathrm{m}^{2}$ ) has reached $32 \%$ among adults aged 20 years and older. ${ }^{1,2}$

The obesity problem in all age groups has been attributed to trends in increasing caloric consumption and decreasing levels of physical activity in the US population over time, as well as to the advent of household technologies that have reduced the time costs of food preparation. ${ }^{3-5}$ Other studies have indicated that there is a substantial familial component to obesity, whether genetic or behavioral in origin. In regional samples, children born to mothers who were obese during pregnancy are dispro- 
portionately likely to be overweight early in childhood, ${ }^{6}$ and children whose parents are obese are more likely to become obese adults themselves. ${ }^{7,8}$ In small community samples, childhood overweight has been associated with grandparental obesity. 8,9

However, cross-generational patterns of obesity have never been reported for a nationally representative sample. In this report, we present the first national analysis of the risk of childhood overweight associated with grandparental obesity, as well as with parental obesity. Our findings illustrate for family physicians and other primary care providers how multigenerational familial patterns of adult obesity can influence the development of childhood overweight.

\section{Methods}

\section{Study Sample}

To examine patterns of BMI among children, their parents, and their grandparents, we used data from the Panel Study of Income Dynamics (PSID), a 35-year panel survey of a nationally representative sample of nearly 8000 US families in 2003, and the Child Development Supplement (CDS), a nationally representative study of children of PSID families. ${ }^{10}$ The PSID has a genealogical design whereby descendants of sample members are themselves interviewed when they begin to live independently. As a result, grandparents of the CDS children (ie, members of the original PSID cohort) are interviewed even when they do not live in the same household as the grandchildren who are part of the CDS cohort.

\section{Data Collection}

Self-reported weight and height information was provided by parents and grandparents in 2003. For children, weight and height information was collected using in-home assessments: a tape measure was used to assess height and a scale was used to measure weight in 2002/2003, when the CDS children were aged 5 to 19 years. Height and weight data were then used to calculate BMI for each respondent, using height and weight data converted to metric measures by the investigators for the BMI calculation.

For children, age- and sex-specific growth curves provided by the Centers for Disease Control and Prevention $(\mathrm{CDC})^{11}$ were used to define BMI categories. By convention, $\mathrm{BMI} \geq 95$ th percentile was defined as overweight, $\mathrm{BMI} \geq 85$ th percentile but $<95$ th percentile was defined as at risk for overweight, $<5$ th percentile was defined as underweight, and otherwise BMI was normal weight. Similarly by CDC convention, for adults BMI $\geq 30$ was defined as obese, BMI 25 to 29.9 was defined as overweight, BMI $<18.5$ was defined as underweight, and otherwise BMI was normal weight.

\section{Data Analysis}

To account for the complex stratified sampling of the PSID and permit inference to the national population, all analyses were sample weighted. Only weighted data are presented, except where otherwise noted.

To compare the PSID sample with the broadly accepted standard national measures of BMI from the National Health and Nutrition Examination Survey (NHANES), we assessed the frequency distribution of children across BMI categories in the PSID measured in 2002/2003 alongside the distribution from NHANES data measured in 1999 to 2002. ${ }^{2}$

To examine the association of child BMI with parental and grandparental BMI, we generated composite measures of adult BMI as follows. For parental BMI, we set the BMI category (underweight, normal weight, overweight, obese) based on mother's BMI and, if missing, used father's BMI. For grandparental BMI, we set the BMI category based on maternal grandmother's BMI and, if missing, used (in order) maternal grandfather's BMI, paternal grandmother's BMI, and paternal grandfather's BMI. This selection rubric was based on analyses that suggest maternal obesity is more strongly determinant of obesity patterns than paternal obesity. ${ }^{6,7}$

With these categories, we used linear regression models to estimate the proportion of children who were overweight in families with different combinations of parental and grandparental BMI categories. We used dummy variables for the different parental and grandparental groups, including missing. We included the association with missing data because it may correspond to clinical situations in which grandparental data are not available because grandparents are deceased or when grandparental weight status is otherwise unknown. We constructed similar models for all children who were either at risk for overweight or overweight (ie, BMI $\geq 85$ th percentile for sex-specific BMI). We do not 
Table 1. Comparison of Body Mass Index Category Distribution Among Children in the Panel Study of Income Dynamics (PSID; 2002/2003) and the National Health and Nutrition Examination Survey (NHANES; 1999-2002)

\begin{tabular}{|c|c|c|c|c|c|c|c|c|c|}
\hline \multirow[b]{2}{*}{ Child's BMI Category } & \multicolumn{3}{|c|}{ Boys and Girls } & \multicolumn{3}{|c|}{ Boys } & \multicolumn{3}{|c|}{ Girls } \\
\hline & PSID (\%) & $\mathrm{n}^{*}$ & NHANES (\%) & PSID (\%) & $\mathrm{n}^{*}$ & NHANES (\%) & PSID (\%) & $\mathrm{n}^{*}$ & NHANES (\%) \\
\hline Underweight & 3.20 & 78 & 3.31 & 3.85 & 41 & 3.60 & 2.57 & 37 & 3.01 \\
\hline Normal & 62.69 & 1580 & 65.06 & 58.78 & 777 & 63.91 & 66.51 & 803 & 66.23 \\
\hline At risk & 15.55 & 400 & 15.14 & 15.69 & 194 & 14.45 & 15.42 & 206 & 15.86 \\
\hline Overweight & 18.55 & 533 & 16.49 & 21.68 & 286 & 18.04 & 15.5 & 247 & 14.9 \\
\hline Total & 100.00 & 2591 & 100 & 100 & 1298 & 100.00 & 100 & 1293 & 100 \\
\hline
\end{tabular}

Percentage is weighted percentage of sample for the PSID or the NHANES. BMI, body mass index.

*Unweighted number of observations in PSID for that category. PSID data are from the Child Development Supplement.

report associations with parental and grandparental underweight because of possible correlation with adults' chronic illness that could not be confirmed in the dataset and because of small sample size for this group that makes estimates unstable.

This study was approved by the University of Michigan Institutional Review Board. Three of the coauthors (KM, RFS, FS) coordinated data collection for the PSID and CDS during the study period.

\section{Results}

\section{Study Sample}

The sample included 2591 children aged 5 to 19 years. Overall, the prevalence of different BMI categories in the 2002/2003 PSID was quite similar to BMI prevalence data from the 1999 to 2002 NHANES (Table 1). Although rates of overweight seemed slightly higher in the PSID than in the NHANES, this is consistent with the fact that the prevalence of overweight has increased rapidly among children in recent years and the PSID data were measured at a later date. ${ }^{1,12}$
We had parental BMI data for 2437 children (94.1\% of sample) and grandparental BMI data for 1573 children ( $60.7 \%$ of sample). The distribution of children in the sample by their parental and grandparental weight status (including missing) appears in Table 2. Among children with known parental and grandparental BMI information, it was most common for children to have normalweight parents and normal-weight grandparents (12.83\% overall), followed by normal-weight parents and overweight grandparents, overweight parents and overweight grandparents, and overweight parents and normal-weight grandparents.

\section{Risk of Childhood Obesity with Parental and Grandparental Obesity}

Among children with known parental and grandparental BMI, the proportion of children overweight for age and sex was $7.9 \%$ among individuals with normal-weight parents and normal-weight grandparents (Table 3, top left-hand corner). This proportion of children who were overweight was less than half of the proportion who were overweight in the sample (18.6\%; Table 1$)$.

Table 2. Distribution of Children Aged 5 to 19 Years by Parental and Grandparental Weight Status

\begin{tabular}{|c|c|c|c|c|c|c|}
\hline & GP Underweight & GP Normal Weight & $\begin{array}{c}\text { GP } \\
\text { Overweight }\end{array}$ & GP Obese & GP Status Missing & Total \\
\hline Parent underweight & $0.07(1)$ & $0.96(17)$ & $1.03(21)$ & $0.60(13)$ & $1.38(25)$ & $4.04(77)$ \\
\hline $\begin{array}{l}\text { Parent normal } \\
\text { weight }\end{array}$ & $0.97(19)$ & $12.83(243)$ & $10.10(230)$ & $5.37(144)$ & $15.42(366)$ & $44.69(1002)$ \\
\hline Parent overweight & $0.14(3)$ & $5.85(112)$ & $6.11(144)$ & $3.49(139)$ & $9.35(277)$ & $24.94(675)$ \\
\hline Parent obese & $0.21(5)$ & $3.06(78)$ & $4.38(113)$ & $5.46(194)$ & $8.30(293)$ & $21.41(683)$ \\
\hline Parent status missing & $0.05(1)$ & $0.46(21)$ & $1.06(27)$ & $1.17(48)$ & $2.17(57)$ & $4.91(154)$ \\
\hline Total & $1.44(29)$ & $18.18(471)$ & $20.65(535)$ & $20.76(538)$ & $39.39(1018)$ & $100(2591)$ \\
\hline
\end{tabular}

Values in table are presented as \% (n). Percentages are weighted; counts are unweighted. GP, grandparent. 
Table 3. Proportion of Children Overweight ( $\geq 95$ th percentile), by Parental and Grandparental Weight Status

\begin{tabular}{lcccc}
\hline & GP Normal Weight & GP Overweight & GP Obese & GP Missing \\
\hline Parent normal weight & $7.9^{*}$ & $7.9^{\dagger}$ & $17.4^{* \ddagger \S}$ & $16.5^{\ddagger}$ \\
Parent overweight & $17.6^{\ddagger}$ & $17.8^{\ddagger}$ & $18.6^{\ddagger}$ & $22.3^{\ddagger}$ \\
Parent obese & $31.9^{\ddagger}$ & $28.3^{\ddagger \ddagger}$ & $29.2^{\ddagger \S}$ & $32.6^{\ddagger}$ \\
\hline
\end{tabular}

Values are presented as percentage. Cases for which parent BMI were not available are not shown. Cases for which child, parent, or grandparent BMI were in underweight category are not shown. GP, grandparent.

${ }^{*}$ Parameters are significantly different from each other at $P<.0001$.

${ }^{\dagger}$ Parameters are significantly different from each other at $P<.0001$.

${ }^{\ddagger}$ Parameter is significantly different from base case group (parent normal and GP normal) at $P<.0001$.

${ }^{\S}$ Parameters are significantly different from each other at $P<.0001$.

Parental and grandparental overweight and obese status had independent associations with childhood overweight. Among children with normal-weight grandparents (Table 3, left-hand column), parental overweight and obese status had a stepwise, statistically significant positive association with children's overweight prevalence. Similarly, among children with normal-weight parents (Table 3 , top row), grandparental obesity was associated with a significantly increased prevalence of children's overweight, compared with families with normal-weight grandparents.

Furthermore, for children with overweight grandparents and with obese grandparents, the presence of parental obesity (but not overweight) was associated with significantly increased prevalence of children's overweight compared with children with normal-weight parents (Table 3). Among children with obese parents, regardless of grandparental weight status, overweight prevalence was almost twice the prevalence rate in the overall sample.

For children whose grandparental BMI data were missing, their probability of overweight corresponded strongly with their parental BMI group. The exception was for children whose parental BMI was normal, but if their grandparental BMI was missing they were significantly more likely to be overweight than if the grandparental BMI was measured as normal $(P<.0001)$.

\section{Comment}

This is the first national study to confirm the association of children's overweight with parental obesity observed in smaller samples ${ }^{6-8}$ and is also the first analysis of a national sample to report an association of children's overweight with grandparental weight status. The positive associations of children's overweight with parental and grandparental obesity in this national sample underscore the continuing clinical and public health importance of familial patterns of obesity in an era when environmental explanations for obesity are receiving considerable research and policy attention. ${ }^{3-5,13}$

Our findings also highlight for family physicians and other primary care practitioners that asking about parental and grandparental patterns of weight can inform the risk of overweight for children. This is important because the majority of children are of normal weight, and it can be difficult for health care providers to anticipate which children are at greatest risk of progressing to overweight later in childhood. These data, although cross-sectional in nature, indicate the relative proportions of a broad age-group of children who may be overweight at some point in childhood, based on their parents' and grandparents' weight characteristics. In the past, many primary care providers may have informally assessed this likelihood of overweight for children in their practice based on parental weight alone. The added insight from these findings is that, for children with normal-weight parents, grandparental obesity is a risk factor for childhood overweight.

Familial effects probably have dual origins in genetic and behavioral mechanisms common to parents and their children. More than 500 genes have been putatively linked to obesity, ${ }^{14}$ and behavior related to dietary preferences and to patterns of physical and sedentary activity are established early in individuals' lifetimes. ${ }^{13}$ Many experts have argued that prevention of obesity during childhood is essential to success of efforts to combat the national obesity epidemic. ${ }^{13,15-17}$ Yet, clinicians and policymakers today have few evidence-based effective tools for obesity prevention among children. ${ }^{13,18}$ 
The strong and independent associations of parental and grandparental obesity with children's overweight in this study lend further imperative to the development of innovative intervention strategies that target young children in their families.

The association of grandparental obesity with childhood overweight, especially in families with normal parental weight, raises additional questions about the intrafamilial mechanisms of obesity. Importantly, although the PSID/CDS includes information about grandparents co-residing with their grandchildren at points in time, it does not include information about duration of co-residence or the grandparents' roles in child care that we believe would be important for an analysis of obesogenic environments. In addition, we were not able to adjust for children's exposure to their grandparents' dietary or physical activity habits, which may have directly influenced children's behaviors. In addition, it is possible that grandparents' behaviors related to their own obesity may have influenced their grandchildren through the children's parents, even if those parents were themselves normal weight. It is also plausible that genetic predisposition to obesity may be variably expressed across generations. More research is warranted to explore these multigenerational effects, especially in clinical situations where children with normal-weight parents may be at increased risk for overweight in the setting of grandparental obesity.

Our findings must be interpreted with important caveats. A chief limitation of this dataset is that adult BMI status was calculated from respondents' self-reported height and weight. Estimates of the prevalence of obesity based on self-report may be affected by bias toward lower BMI from underreporting of weight influenced by social desirability. ${ }^{19,20}$ Of note, self-report adult BMI data from the PSID closely match other self-report data available from concurrent waves of the National Health Interview Survey. ${ }^{21}$ Another limitation is that we assessed intrafamilial BMI associations at one point in time; future work will permit assessments of these relationships over longer periods.

Last, we had BMI data for grandparents for approximately $60 \%$ of the children's sample, which largely reflects survival status of the grandparents rather than item nonresponse on the height and weight questions. Importantly, children with normal-weight parents were at increased risk if their grandparental BMI data were missing, raising the possibility that deceased grandparents had been obese and perhaps died prematurely related to health consequences of obesity such as coronary heart disease and diabetes. Information that clinicians can collect from families regarding deceased grandparents' weight status may well be more complete and informative than data available for this analysis.

\section{Conclusion}

Overall, these findings suggest that overweight among children is strongly associated with patterns of obesity not only among their parents but also among their grandparents. Whether these patterns are related most strongly to behaviors, genetics, or today's obesogenic environment, the imperative remains to address children's weight status where children consume a large share of their calories with their families. Primary care physicians may find it productive to engage families in discussions about generational patterns of weight, as both a window on children's risk of overweight and an opportunity to discuss familial patterns of diet and physical activity that can affect the health of multiple generations.

\section{References}

1. Ogden CL, Carroll MD, Flegal KM. High body mass index for age among US children and adolescents, 2003-2006. JAMA 2008;299:2401-5.

2. Ogden CL, Carroll MD, Curtin LR, et al. Prevelance of overweight and obesity in the United States, 1999-2004. JAMA 2006;295:1549-55.

3. Costa DL, Steckel RH. Long-term trends in health, welfare, and economic growth in the United States (November 1995). NBER Working Paper No. H0076. Available at: http://papers.ssrn.com/sol3/ papers.cfm?abstract_id=190415. Accessed 5 September 2008.

4. Philipson TJ, Posner RA. The long-run growth in obesity as a function of technological change. NBER Working Paper No. 7423. 1999. Available at http:// www.nber.org/papers/w7423. Accessed 5 April 2007.

5. Cutler DM, Glaeser EL, Shapiro JM. Why have Americans become more obese? Harvard Institute of Economic Research, Discussion Paper No. 1994. 2003. Available at: http://papers.ssrn.com/sol3/ papers.cfm?abstract_id $=370430$. Accessed 5 September 2008.

6. Whitaker R. Predicting preschooler obesity at birth: the role of maternal obesity in early pregnancy. Pediatrics 2004;114:e29-36.

7. Whitaker RC, Wright JA, Pepe MS, Seidel KD, Dietz WH. Predicting obesity in young adulthood 
from childhood and parental obesity. N Engl J Med 1997;337:869-73.

8. Garn SM, LaVelle M. Two-decade follow-up of fatness in early childhood. Am J Clin Nutr 1981;34: $148-53$.

9. Polley DC, Spicer MT, Knight AP, Hartley BL. Intrafamilial correlates of overweight and obesity in African-American and Native-American grandparents, parents, and children in rural Oklahoma. J Am Diet Assoc 2005;105:262-5.

10. Institute of Social Research, University of Michigan. Panel Study of Income Dynamics, Child Development Supplement. Available at: http://psidonline.isr.umich.edu/CDS/cdsguide.html. Accessed 5 April 2007.

11. Centers for Disease Control and Prevention. Growth charts. Available at: http://www.cdc.gov/ growthcharts/. Accessed 5 September 2008.

12. Ogden CL, Flegal KM, Carroll MD, Johnson CL. Prevalence and trends in overweight among US children and Adolescents, 1999-2000. JAMA 2002;288: $1728-32$.

13. Koplan JP, Liverman CT, Kraak VI, Institute of Medicine (U.S.). Committee on Prevention of Obesity in Children and Youth, Institute of Medicine (U.S.). Board on Health Promotion and Disease Prevention. Preventing childhood obesity: health in the balance. Washington, DC: National Academies Press; 2005.
14. Rankinen T, Zuberi A, Chagnon YC, et al. The human obesity gene map: the 2005 update. Obesity 2006;14:529-644.

15. Dietz WH. Critical periods in childhood for the development of obesity. Am J Clin Nutr 1994;59:955-9.

16. Barlow SE, Dietz WH. Obesity evaluation and treatment: expert committee recommendations. Pediatrics 1998;102:e29. Available at: http://www.pediatrics.org/cgi/content/full/102/3/e29. Accessed 5 April 2007.

17. Dietz WH, Robinson TN. Overweight children and adolescents. N Engl J Med 2005;352:2100-9.

18. Summerbell CD, Waters E, Edmunds LD, Kelly S, Brown T, Campbell KJ. Interventions for preventing obesity in children. Cochrane Database Syst Rev 2005;20:CD001871.

19. Black DR, Taylor AM, Coster DC. Accuracy of self-reported body weight: Stepped Approach Model component assessment. Health Educ Res 1998;13: 301-7.

20. Jeffery RW. Bias in reported body weight as a function of education, occupation, health and weight concern. Addict Behav 1996;21:217-22.

21. Andreski P, Gouskova E, McGonagle K, Schoeni B. An analysis of the quality of the health data in the Panel Study of Income Dynamics: 1999-2003. Available at: http://psidonline.isr.umich.edu/Guide/ Quality/report_on_health_qsv2.pdf. Accessed 5 September 2008. 